\title{
Bacteriemia por Rothia mucilaginosa en niños inmunocomprometidos: reporte de casos y revisión de la literatura
}

\author{
Rothia mucilaginosa bacteremia in immunocompromised \\ children: case report and review of the literature
}

\author{
José Iván Castillo Bejarano,* Samantha Pérez Cavazos,* Daniela Cisneros Saldaña,* \\ Erika Aidé Larragoity González, ${ }^{*}$ Fernando Espinosa Villaseñor,* \\ María Mayela Rodríguez Saldívar,* Eduardo Romo Leija,* Gilberto De la Peña Aguilar,* \\ Denisse Natalie Vaquera Aparicio,* Abiel Homero Mascareñas de los Santos* \\ * División de Infectología Pediátrica, Departamento de Pediatría del Hospital Universitario «Dr. José \\ Eleuterio González», Universidad Autónoma de Nuevo León. México.
}

\section{RESUMEN}

\begin{abstract}
Rothia mucilaginosa, previamente conocido como Stomatococcus mucilaginosus, es un cocobacilo Gram-positivo que forma parte de la microbiota de cavidad oral y vías respiratorias superiores. Las infecciones por este microorganismo son infrecuentes, presentándose principalmente en niños inmunocomprometidos. Actualmente es reconocido como un patógeno oportunista, y emergente en niños con fibrosis quística. En el presente trabajo describimos dos casos de pacientes pediátricos oncológicos con bacteriemias primarias por $R$. mucilaginosa; además, se realizó una revisión de la literatura de bacteriemias por $R$. mucilaginosa en niños inmunocomprometidos.
\end{abstract}

Palabras clave: Bacteriemia, inmunocompromiso, leucemia linfoblástica aguda, Rothia spp, Rothia mucilaginosa.

\section{INTRODUCCIÓN}

Rothia mucilaginosa es una bacteria Gram-positiva, cocobacilo ovoide, encapsulada, no formadora de esporas, no móvil, que se puede agrupar en pares, tríadas, tétradas o racimos; además, se considera aerobia o anaerobia facultativo. Previamente denominada Stomatococcus mucilaginosus o Micrococcus

\section{ABSTRACT}

Rothia mucilaginosa, previously known as Stomatococcus mucilaginosus, is a Gram-positive coccobacillus that is part of the microbiota of the oral cavity, and upper respiratory tract. Infections with this microorganism are infrequent, occurring mainly in immunocompromised children. It is currently recognized as an opportunistic pathogen and emerging in children with cystic fibrosis. In the present work, we describe two cases of pediatric oncology patients with primary R. mucilaginosa bacteremia, and a literature review of $\mathrm{R}$. mucilaginosa bacteremia in immunocompromised children.

Keywords: Bacteremia, immunocompromised, acute lymphoblastic leukemia, Rothia spp, Rothia mucilaginosa.

mucilaginosus, se reclasifica al género Rothia spp. en el año 2000, con base en su asociación filogenética específica con Rothia dentocariosa. ${ }^{1-11}$ El género Rothia se encuentra dentro de la familia Micrococcaceae e incluye siete especies: $R$. dentocariosa, $R$. mucilaginosa, $R$. aeria, $R$. nasimurium, $R$. amarae, $R$. endophytica y $R$. terrae, de las cuales, las primeras tres forman parte de la microbiota de boca y tracto

Citar como: Castillo BJI, Pérez CS, Cisneros SD, Larragoity GEA, Espinosa VF, Rodríguez SMM et al. Bacteriemia por Rothia mucilaginosa en niños inmunocomprometidos: reporte de casos y revisión de la literatura. Rev Latin Infect Pediatr. 2021; 34 (4): 193-202. https:// dx.doi.org/10.35366/102970

Recibido: 12-10-2021. Aceptado: 15-10-2021.

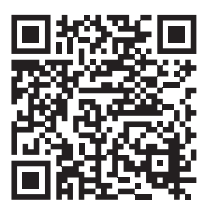


respiratorio superior, y se han descrito como agentes etiológicos de enfermedad periodontal y caries en personas inmunocompetentes, así como causa de enfermedad invasiva en contexto de inmunocompromiso. ${ }^{1,2,4,8,11-14}$

La primera infección reportada por $R$. mucilaginosa fue en 1978, y se trató de una endocarditis infecciosa tras un procedimiento de cateterización cardiaca, y desde entonces se han identificado infecciones invasivas predominantemente en personas inmunocomprometidas. ${ }^{7,15,16}$ En este trabajo describimos las características clínicas y desenlace de dos casos de bacteriemia primaria por $R$. mucilaginosa en niños de un centro hospitalario de tercer nivel en México; además, se realiza revisión de la literatura de casos reportados en niños inmunocomprometidos.

\section{CASOS CLÍNICOS}

Caso clínico 1: femenina de cinco años con diagnóstico de leucemia linfoblástica aguda de células $B$ en fase de inducción y esquema antibiótico profiláctico con ciprofloxacino y trimetoprim con sulfametoxazol. Recibió su última quimioterapia (QT) 24 horas previas a su ingreso con vincristina y pegaspargasa. Acudió a nuestra institución por fiebre de $38^{\circ} \mathrm{C}$ sin otro síntoma acompañante. A la exploración física presenta mucositis oral grado I de la OMS (Organización Mundial de la Salud), sin evidencia de foco infeccioso. Se inició protocolo institucional de fiebre y neutropenia con toma de laboratorios y cultivos, en donde se documentó neutropenia profunda con recuento absoluto de neutrófilos 0 células $/ \mathrm{mm}^{3}$. Se inició manejo empírico con meropenem (60 mg/kg/día) intravenoso. A las 24 horas persistía febril y se reportó crecimiento de cocos Gram-positivos de dos hemocultivos periféricos, por lo que se agregó vancomicina $(60 \mathrm{mg} / \mathrm{kg} /$ día). A las 72 horas se identificó $R$. mucilaginosa mediante espectrofotometría de masas con tiempo de vuelo (MALDI-TOF MS) sensible a vancomicina y linezolid, intermedio a eritromicina, y resistente a clindamicina, levofloxacino, oxacilina, trimetoprim con sulfametoxazol y penicilina. La paciente presentó evolución favorable, con resolución de la fiebre, remisión de neutropenia severa, negativización de cultivos, luego de completar siete días de meropenem y 12 días de vancomicina intravenosa, se egresó sin presentarse complicaciones con seguimiento ambulatorio de cuatro meses.
Caso clínico 2: masculino de dos años con hepatoblastoma epitelial PRETEXT (pre-treatment extent) 3 estadio III, diagnosticado al año, con resección tumoral y QT adyuvante. Recibió doxorrubicina y cisplatino nueve días previos a su ingreso. Acudió a urgencias por presentar síndrome purpúricoanémico, y un pico febril de $38.5^{\circ} \mathrm{C}$. A la exploración física con mucositis oral grado I de la OMS. Se inició protocolo de neutropenia febril con toma de cultivos de sangre, orina y administración de imipenemcilastatina $(60 \mathrm{mg} / \mathrm{kg} / \mathrm{día}$ ). Se reportó crecimiento de cocos Gram-positivos a las 14 y 15 horas de dos hemocultivos periféricos, por lo que se agregó vancomicina (60 mg/kg/día) al manejo. Después se identificó R. mucilaginosa por MALDI-TOF MS, sensible a eritromicina, meticilina, levofloxacino, ciprofloxacino, intermedio a clindamicina; resistente a penicilina y ampicilina. Luego de dos días de vancomicina, el paciente persiste febril, por lo que se suspendió el carbapenémico y se agregó levofloxacino (IV, 20 mg/ kg/día); además, se solicitaron nuevos hemocultivos, que evidenciaron la persistencia de $R$. mucilaginosa. A las 72 horas del inicio del levofloxacino cedió la fiebre, y se egresó el paciente tras cumplir esquema con siete días vancomicina y 10 de levofloxacino, con buena evolución clínica, resolución de neutropenia, y negativización de hemocultivos luego de 48 horas de inicio de levofloxacino.

\section{DISCUSIÓN}

El género Rothia se considera por el Instituto de Estándares para el Laboratorio Clínico (CLSI, por sus siglas en inglés) como un microorganismo fastidioso y de aislamiento infrecuente. ${ }^{4,17}$ Crece de forma adecuada en agar sangre, en aerobiosis o en ambiente enriquecido con $\mathrm{CO}_{2}$, y no reporta crecimientos en medios con $\mathrm{NaCl} \geq 5 \%$. Las colonias se visualizan convexas, blanquecinas, mucoides, con $\gamma$-hemólisis, a partir de las 24 horas de incubación. La identificación mediante pruebas bioquímicas arroja un microorganismo catalasa variable, oxidasa negativo, con hidrolisis de esculina y gelatina. $3,7,9,10,15,17,18$

Para la realización de las pruebas de susceptibilidad a los antimicrobianos se debe cultivar en agar líquido de ajuste catiónico Mueller Hinton suplementado con sangre lisada de caballo a temperatura ambiente, en el cual se puede observar el crecimiento de colonias en un rango de 20 a 24 horas. Los medicamentos que se sugieren probar de 
Rev Latin Infect Pediatr. 2021; 34 (4): 193-202

primera instancia para corroborar susceptibilidad son penicilina y vancomicina. ${ }^{17}$

El espectro de síndromes clínicos causados por R. mucilaginosa en pacientes inmunocomprometidos incluye endocarditis, meningitis, neumonía, bacteriemia, periodontitis, osteomielitis, artritis séptica, infección de piel y tejidos blandos, infección de dispositivos protésicos y endoftalmitis. ${ }^{1,3,10,13}$ Los factores de riesgo más importantes para el desarrollo de infecciones invasivas por este microorganismo son la presencia de enfermedades hematooncológicas, neutropenia severa, neutropenia prolongada, uso de dispositivos intravasculares, sondas a permanencia, dispositivos protésicos, mucositis y esteroides a altas dosis. $3,5,11,13$

Se realizó la búsqueda de la literatura en las bases de datos Medline (US National Library of Medicine [NLM]), SciELO (Scientific Electronic Library Online), LILACS (Latin American and Caribbean Literature in Health Science), Scopus (Elsevier, Amsterdam, Netherland), Excerpta Medica Database (Embase [Elsevier, Amsterdam, Netherlands]) y Cumulative Index to Nursing and Allied Health Literature (CINAHL [EBSCO, Ipswich, Massachusetts]), Europe PMC (European Bioinformatics Institute), Web of Science (Clarivate Analytics, Philadelphia, Pennsylvania) y Google Scholar (Google, Palo Alto, California). La estrategia de búsqueda se desarrolló con el uso de palabras clave «Rothia spp», «bacteremia», «Rothia mucilaginosa», "Stomatococcus mucilaginosus», «immunocompromised», "pediatric patient», "children», "cancer», "malignancy» y «leukemia». Ver estrategia de búsqueda en el Anexo 1. La búsqueda incluyó los estudios publicados a partir del 1 de enero de 1990 a la fecha.

En esta revisión se incluyeron los estudios en inglés y español que cumplieron con los siguientes criterios: 1) estudios observacionales publicados entre enero de 1990 a mayo de 2021 ; 2) estudios observacionales bacteriemia confirmada por $R$. mucilaginosa en niños con inmunodeficiencias primarias o secundarias. Se incluyeron reportes de caso, series de caso, cartas al editor, que documentaran bacteriemia por $R$. mucilaginosa en niños, con o sin complicaciones infecciosas, como meningitis, infecciones de piel y tejidos blandos, infección asociada a catéter venoso central, endocarditis, neumonía, sepsis y choque séptico. Se consideró como niños aquéllos con edad entre cero a 18 años. Se analizaron las referencias de las publicaciones con la finalidad de identificar casos adicionales o repetidos.
Se excluyeron del análisis aquellos reportes que sólo incluyeran casos de adultos, niños inmunocompetentes, o niños inmunocomprometidos con datos individuales incompletos, o casos con infección por R. mucilaginosa sin bacteriemia identificada.

Se encontraron 306 artículos, de los cuales 15 cumplieron los criterios de inclusión (Figura 1). En total se identificaron 29 casos de bacteriemia por $R$. mucilaginosa en niños inmunocomprometidos, y se analizaron en conjunto con los dos casos reportados en este trabajo (Tabla 1). La media de edad fue 7.2 años (rango de cero a 17 años), con predominio de sexo masculino con 22 casos (71\%). Del total, 21 niños (67\%) padecían algún tipo de malignidad hematológica y siete niños (23\%) tumores sólidos malignos (Tabla 2). Hasta la fecha, esta revisión reúne el mayor número de bacteriemias por $R$. mucilaginosa en niños inmunocomprometidos $(\mathrm{n}=$ 31), previamente Chavan y colaboradores reportaron una serie de 36 casos de infección, de los cuales 32 eran niños, y sólo 26 eran bacteriemias. ${ }^{9}$

En 19 pacientes (61\%) se reportó la administración de un tratamiento que condiciona a inmunosupresión, de los cuales 18 (58\%) se encontraban bajo tratamiento antineoplásico, tres con esteroide (10\%) y dos con radioterapia (6\%).

Otros factores de riesgo analizados identificaron la presencia de neutropenia como factor más frecuente, similar a otros reportes en los cuales se documenta neutropenia en 85 a 100\% de los casos de infecciones por Rothia spp. ${ }^{5,15}$ En nuestro análisis se reportó una alta incidencia de neutropenia severa $(n=21[68 \%])$, de los cuales 11 (35\%) tenían neutropenia profunda, y sólo $6 \%$ de los casos $(n=2)$ presentaron conteo absoluto de neutrófilos dentro de la normalidad. En ocho casos (26\%) no se especificó el recuento de neutrófilos absoluto. En cambio, la presencia de mucositis, la cual se considera un portal de entrada para bacteriemia, se encontró en menos de $29 \%$ de los niños ( $n=9 ; 29 \%)$, mientras que series de casos menores con infecciones por Rothia spp, como la reportada por Henwick y Wang se documentó en cerca de $50 \%$. 5,15

La presencia de dispositivos intravasculares centrales también se presentó en $68 \%$ de los casos, lo cual coincide con la importancia de estos dispositivos como factor de riesgo, ya que en otros casos se reporta hasta $81 \%$ el uso de catéteres intravasculares. ${ }^{15}$ Ramos y colaboradores reportaron cinco pacientes con bacteriemia, de los cuales cuatro eran usuarios de catéteres protésicos, como venoso, 


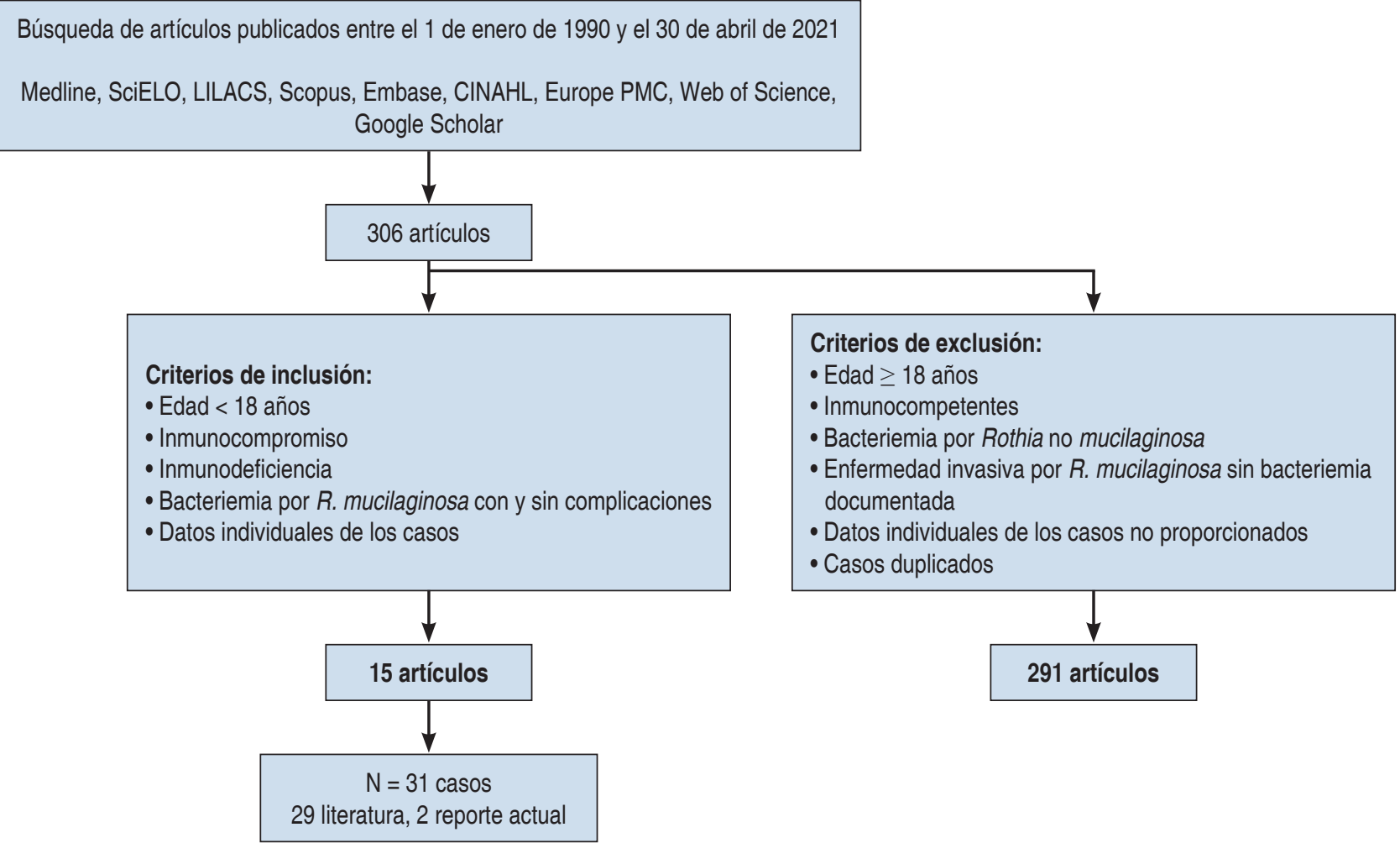

Figura 1: Flujograma de estrategia de búsqueda.

urinario o biliar. Debido a que la bacteria posee la capacidad de formación de biopelículas en dispositivos, se facilita la adhesión de microorganismos en el dispositivo y dificulta la penetración del antibiótico en este elemento, y pudiera provocar manifestaciones sistémicas como émbolos sépticos. De tal forma que la presencia de los dispositivos representa un factor de riesgo para el desarrollo de bacteriemia por $R$. mucilaginosa, fallas terapéuticas en ausencia de la remoción del dispositivo. . $^{14,19,20}$

En 19 niños (61\%) se documentaron 30 eventos de complicaciones relacionadas con bacteriemia por R. mucilaginosa, siendo neumonía la más prevalente $(n=9 ; 29 \%)$, seguido de infección asociada a catéter $(n=5 ; 16 \%)$, meningitis $(n=5 ; 16 \%)$ y choque séptico $(n=5 ; 16 \%)$. En series de casos de niños con bacteriemia por $R$. mucilaginosa se han reportado complicaciones como meningitis, neumonía e insuficiencia respiratoria, hasta en un $45 \%$ de los casos. ${ }^{9,11}$ Chavan y colaboradores, en su serie de 11 casos describe en cuatro pacientes $(36 \%)$ el desarrollo de complicaciones en sistema nervioso central, a pesar del tratamiento de erradica- ción previo. ${ }^{9}$ En otros estudios de meningitis por $R$. mucilaginosa, se logró identificar bacteriemia hasta en $62 \%$ de los casos. ${ }^{21}$ En series como la reportada por Henwick y colaboradores, se documentó presencia de complicaciones en ocho de nueve pacientes, como fiebre persistente, neumonía, síndrome de insuficiencia respiratoria aguda pediátrica, choque séptico, ectima gangrenoso, meningitis y celulitis. ${ }^{15}$ Los reportes previos sugieren la búsqueda intencionada de complicaciones como choque, neumonía y meningitis, en todos los casos con bacteriemia por R. mucilaginosa.

Debido a la escasez de casos documentados de bacteriemia por $R$. mucilaginosa, no se ha establecido el tratamiento de primera línea. ${ }^{9,17}$ Tampoco se han establecido pautas de duración de tratamiento, y autores como Bayhan y colaboradores, recomiendan esquemas de entre siete y 21 días, basado en la respuesta clínica y la presencia de comorbilidades. $9,13,19$

En cuanto al tratamiento administrado, se empleó vancomicina en $97 \%$ de los casos; sin embargo, no se logró establecer en todos los casos si se administró 
Tabla 1: Resumen de los factores de riesgo, tratamiento y desenlace de bacteriemias por Rothia mucilaginosa en niños inmunocomprometidos.

\begin{tabular}{|c|c|c|c|c|c|c|c|c|c|c|}
\hline $\begin{array}{l}\text { Año y } \\
\text { lugar de } \\
\text { publicación }\end{array}$ & $\begin{array}{l}\text { Edad/ } \\
\text { género }\end{array}$ & $\begin{array}{l}\text { Enfermedad de } \\
\text { base }\end{array}$ & $\begin{array}{c}\text { Estado de } \\
\text { inmunocompromiso }\end{array}$ & Complicaciones & $\begin{array}{l}\text { Neutrófilos } \\
\text { totales } / \mathrm{mm}^{3}\end{array}$ & Mucositis & $\begin{array}{l}\text { Portador } \\
\text { de CVC }\end{array}$ & Tratamiento empírico & $\begin{array}{l}\text { Días de } \\
\text { antibiótico }\end{array}$ & $\begin{array}{l}\text { Desenlace/ } \\
\text { respuesta al } \\
\text { tratamiento }\end{array}$ \\
\hline 1990/EUA ${ }^{22}$ & $\operatorname{Sin}_{\text {datos } / M}$ & LLA recaída & QT mantenimiento & No & NE & No & Sí & $\begin{array}{l}\text { Vancomicina, } \\
\text { ceftazidima y } \\
\text { tobramicina }\end{array}$ & 14 & Vivo \\
\hline 1991/EUA ${ }^{33}$ & $13 / F$ & $\begin{array}{l}\text { Sarcoma } \\
\text { osteogénico }\end{array}$ & $\begin{array}{l}\text { QT cisplatino y } \\
\text { doxorrubicina }\end{array}$ & $\begin{array}{l}\text { Infección asociada } \\
\text { con CVC }\end{array}$ & 0 & No & Sí & $\begin{array}{c}\text { Vancomicina y } \\
\text { ceftazidima, } \\
\text { cambió a cefazolina }\end{array}$ & $\begin{array}{l}21 \text { total } \\
(4 \text { y } 17)\end{array}$ & Viva \\
\hline \multirow{3}{*}{$1991 / E^{2} A^{23}$} & $2.5 / \mathrm{M}$ & $\begin{array}{l}\text { Tumor del seno } \\
\text { endodérmico }\end{array}$ & NE & $\begin{array}{l}\text { Neumonía } \\
600 \text { WBC }\end{array}$ & NE & NE & Sí & $\begin{array}{l}\text { Vancomicina y } \\
\text { ceftazidima }\end{array}$ & 11 & Vivo \\
\hline & $4 / \mathrm{M}$ & $\begin{array}{l}\text { Leucemia no } \\
\text { especificada }^{a}\end{array}$ & NE & No & 4,800 WBC & NE & Sí & Vancomicina & 21 & Vivo \\
\hline & $9 / M$ & Neuroblastoma & NE & $\begin{array}{c}\text { Infección asociada } \\
\text { a CVC }\end{array}$ & $<500$ & NE & Sí & Vancomicina & 14 & Vivo \\
\hline 1992/EUA ${ }^{24}$ & O/M & $\begin{array}{c}\text { Prematurez } \\
\text { extrema (28 SDG), } \\
\text { RPM }\end{array}$ & Dexametasona & Meningitis & $\begin{array}{c}\mathrm{NE} \\
12,000 \mathrm{WBC}\end{array}$ & No & Sí & $\begin{array}{l}\text { Vancomicina y } \\
\text { gentamicina }\end{array}$ & 24 & Vivo \\
\hline \multirow{6}{*}{ 1993/EUA ${ }^{15}$} & $7 / F$ & Neuroblastoma & NE & $\begin{array}{c}\text { Infección } \\
\text { asociada a CVC }\end{array}$ & $<500$ & No & Sí & Vancomicina & NE & Vivo \\
\hline & $10 / M$ & LMA remisión & NE & $\begin{array}{c}\text { Neumonía } \\
\text { Choque séptico }\end{array}$ & $<500$ & No & Sí & Vancomicina & NE & Vivo \\
\hline & $1.8 / \mathrm{F}$ & $\begin{array}{l}\text { LMA y síndrome } \\
\text { de Down }\end{array}$ & NE & No & $<500$ & No & Sí & Vancomicina & NE & Vivo \\
\hline & $16 / M$ & LLA-T recaídac & QT reinducción & $\begin{array}{c}\text { Neumonía } \\
\text { Choque séptico }\end{array}$ & 0 & NE & NE & $\begin{array}{l}\text { Vancomicina y } \\
\text { ceftazidima; cambió } \\
\text { a clindamicina y } \\
\text { amikacina }\end{array}$ & 24 & Vivo \\
\hline & $17 / F$ & $\begin{array}{l}\text { LMA y post-TCHP } \\
\text { alogénico }\end{array}$ & $\begin{array}{l}\text { QT citarabina, } \\
\text { ciclofosfamida, RT }\end{array}$ & $\begin{array}{c}\text { Neumonía } \\
\text { Choque séptico } \\
\text { Meningitis } \\
\text { Sepsis relacionada } \\
\text { a catéter } \\
\text { Ectima gangrenoso }\end{array}$ & 18 & Sí & Sí & $\begin{array}{l}\text { Vancomicina y } \\
\text { ceftazidima; se } \\
\text { suspendió } \\
\text { ceftazidima, se } \\
\text { agregó ceftriaxona } \\
\text { y se proporcionó } \\
\text { vancomicina intratecal } \\
\text { en dosis única }\end{array}$ & NE & Vivo \\
\hline & $2 / F$ & LLA & QT inducción & $\begin{array}{l}\text { Neumonía } \\
\text { Celulitis }\end{array}$ & $<500$ & Sí & No & Vancomicina & NE & Vivo \\
\hline
\end{tabular}


Continúa la Tabla 1: Resumen de los factores de riesgo, tratamiento y desenlace de bacteriemias por Rothia mucilaginosa en niños inmunocomprometidos.

\begin{tabular}{|c|c|c|c|c|c|c|c|c|c|c|}
\hline $\begin{array}{l}\text { Año y } \\
\text { lugar de } \\
\text { publicación }\end{array}$ & $\begin{array}{l}\text { Edad/ } \\
\text { género }\end{array}$ & $\begin{array}{l}\text { Enfermedad de } \\
\text { base }\end{array}$ & $\begin{array}{c}\text { Estado de } \\
\text { inmunocompromiso }\end{array}$ & Complicaciones & $\begin{array}{l}\text { Neutrófilos } \\
\text { totales } / \mathrm{mm}^{3}\end{array}$ & Mucositis & $\begin{array}{l}\text { Portador } \\
\text { de CVC }\end{array}$ & Tratamiento empírico & $\begin{array}{c}\text { Días de } \\
\text { antibiótico }\end{array}$ & $\begin{array}{l}\text { Desenlace/ } \\
\text { respuesta al } \\
\text { tratamiento }\end{array}$ \\
\hline & $8 / M$ & LMA remisión & NE & No & $<500$ & Sí & Sí & Vancomicina & NE & Vivo \\
\hline & $3 / M$ & LMA remisión & NE & Choque séptico & $<500$ & No & Sí & Vancomicina & NE & Vivo \\
\hline & $4 / F$ & $\begin{array}{l}\text { Rabdomiosarcoma } \\
\text { en remisión }\end{array}$ & NE & No & No & No & No & Vancomicina & NE & Vivo \\
\hline $1993 /$ USA $^{28}$ & $10 / \mathrm{M}$ & $\begin{array}{l}\text { LLA células pre-B } \\
\text { en recaída }\end{array}$ & QT reinducción & $\begin{array}{c}\text { Infección de vías } \\
\text { urinarias con } \\
\text { urocultivo }+R \text {. } \\
\text { mucilaginosa }\end{array}$ & $\begin{array}{c}\text { NE } \\
900 \text { WBC }\end{array}$ & NE & No & $\begin{array}{l}\text { Vancomicina y } \\
\text { ceftazidima }\end{array}$ & 1 & $\begin{array}{c}\text { Muerto/ } \\
\text { diagnóstico } \\
\text { post mortem }\end{array}$ \\
\hline 1994/Suecia ${ }^{26}$ & $10 / \mathrm{M}$ & $\begin{array}{c}\text { Adrenoleucodistrofia } \\
+ \text { TCHPd }^{d}\end{array}$ & $\begin{array}{l}\text { QT busulfán y } \\
\text { ciclofosfamida } \\
\text { Profilaxis: } \\
\text { ciclosporina, } \\
\text { metotrexate }\end{array}$ & Neumonía & $\begin{array}{l}\text { Neutropenia } \\
\text { NE } \\
\text { Se encontraba } \\
\text { en día }+10 \text { de } \\
\text { trasplante } \\
\text { al momento del } \\
\text { aislamiento. } \\
\text { Aún sin } \\
\text { prendimiento }\end{array}$ & Sí & $\mathrm{NE}$ & $\begin{array}{l}\text { Imipenem-cilastatina y } \\
\text { vancomicina, y } \\
\text { anfotericina B } \\
\text { liposomal }\end{array}$ & $\mathrm{NE}$ & NE \\
\hline 1997/EUA ${ }^{12}$ & $5 / M$ & LLA & NE & $\begin{array}{c}\text { Meningitis } R \text {. } \\
\text { mucilaginosa } 6 \\
\text { semanas después } \\
\text { de bacteriemia }\end{array}$ & $\begin{array}{l}\text { Neutropenia } \\
\text { valor } \mathrm{NE}\end{array}$ & No & Sí & $\begin{array}{l}\text { Vancomicina, } \\
\text { ceftazidima }^{a}\end{array}$ & 7,17 & Vivo \\
\hline 1998/España ${ }^{8}$ & $16 / \mathrm{M}$ & LLA recaída & $\begin{array}{c}\text { Reinducción: } \\
\text { prednisona, } \\
\text { citarabina, } \\
\text { mitoxantrona, etopósido }\end{array}$ & No & NE & Sí & Sí & $\begin{array}{c}\text { Amikacina, imipenem, } \\
\text { y anfotericina B, } \\
\text { posteriormente se } \\
\text { agrega vancomicina }\end{array}$ & 21 & Vivo \\
\hline 2007/ltalia ${ }^{27}$ & $3 / \mathrm{M}$ & $\begin{array}{l}\text { Síndrome de } \\
\text { Shwachman- } \\
\text { Diamond }\end{array}$ & NE & No & 6,734 WBC & No & No & $\begin{array}{l}\text { Ampicilina/sulbactam } \\
\text { y netilmicina; } \\
\text { posteriormente se } \\
\text { cambia a rifampicina }\end{array}$ & $\begin{array}{c}\text { Ampicilina/ } \\
\text { sulbactam } 5 \\
\text { días; } \\
\text { rifampicina } \\
10 \\
\end{array}$ & Vivo \\
\hline \multirow[t]{3}{*}{ 2013/EUA ${ }^{9}$} & $14 / \mathrm{M}$ & LLA & NE & No & $<500$ & No & NE & Vancomicina & NE & Muerto \\
\hline & $12 / \mathrm{M}$ & $\begin{array}{l}\text { LLA células B } \\
\text { recaída, } \\
\text { post-TCHP }\end{array}$ & QT reinducción & $\begin{array}{c}\text { Neumonía } \\
\text { complicada } \\
\text { Choque séptico }\end{array}$ & 0 & No & Sí & $\begin{array}{l}\text { Vancomicina, } \\
\text { ticarcilina-clavulanato, } \\
\text { gentamicina }\end{array}$ & 3 & Muerto \\
\hline & $7 / F$ & LLA células pre-Be & QT reinducción & $\begin{array}{c}\text { Infección asociada } \\
\text { a CVC } \\
\text { Meningitis }\end{array}$ & 0 & No & Sí & $\begin{array}{l}\text { Vancomicina, } \\
\text { ceftazidima } \\
\text { Posteriormente } \\
\text { desarrolla meningitis } \\
\text { por } R \text { mucilaginosa } \\
\text { y se trata con } \\
\text { vancomicina } \\
\text { cefotaxima, } \\
\text { rifampicina } 21 \text { días }\end{array}$ & 21 & Vivo \\
\hline
\end{tabular}


Continúa la Tabla 1: Resumen de los factores de riesgo, tratamiento y desenlace de bacteriemias por Rothia mucilaginosa en niños inmunocomprometidos.

\begin{tabular}{|c|c|c|c|c|c|c|c|c|c|c|}
\hline $\begin{array}{l}\text { Año y } \\
\text { lugar de } \\
\text { publicación }\end{array}$ & $\begin{array}{l}\text { Edad/ } \\
\text { género }\end{array}$ & $\begin{array}{l}\text { Enfermedad de } \\
\text { base }\end{array}$ & $\begin{array}{c}\text { Estado de } \\
\text { inmunocompromiso }\end{array}$ & Complicaciones & $\begin{array}{l}\text { Neutrófilos } \\
\text { totales } / \mathrm{mm}^{3}\end{array}$ & Mucositis & $\begin{array}{l}\text { Portador } \\
\text { de CVC }\end{array}$ & Tratamiento empírico & $\begin{array}{l}\text { Días de } \\
\text { antibiótico }\end{array}$ & $\begin{array}{l}\text { Desenlace/ } \\
\text { respuesta al } \\
\text { tratamiento }\end{array}$ \\
\hline & $4 / \mathrm{M}$ & $\begin{array}{l}\text { LMA recaída, post- } \\
\text { TCHP } 2 \text { ocasiones }\end{array}$ & $\begin{array}{l}\text { QT hidroxiurea, } \\
\text { etopósido, prednisona }\end{array}$ & $\begin{array}{l}\text { Sepsis } \\
\text { Neumonía }\end{array}$ & 0 & NE & Sí & $\begin{array}{l}\text { Vancomicina, } \\
\text { ticarcilina-clavulanato, } \\
\text { gentamicina }\end{array}$ & 4 & Muerto \\
\hline 2016/Turquía ${ }^{13}$ & $4 / M$ & $\begin{array}{l}\text { Neuroblastoma IV, } \\
\text { recaída a SNC, } \\
\text { resección de } \\
\text { glándula suprarrenal } \\
\text { derecha, } \\
\text { post-TCHP } \\
\text { autólogo }\end{array}$ & $\mathrm{QT}+\mathrm{RT}$ & Neumonía & No & No & No & $\begin{array}{l}\text { Vancomicina, } \\
\text { meropenem }\end{array}$ & 14 & Vivo \\
\hline 2016/Japón ${ }^{10}$ & $4 / \mathrm{M}$ & $\begin{array}{l}\text { LLA, síndrome de } \\
\text { Down }\end{array}$ & QT reinducción & No & 7 & Sí & Sí & $\begin{array}{l}\text { Vancomicina, } \\
\text { meropenem }\end{array}$ & 21 & Vivo \\
\hline \multirow[t]{2}{*}{ 2020/Chile ${ }^{11}$} & $10 / F$ & LLA células T & $\begin{array}{l}\text { QT fase de } \\
\text { consolidación }\end{array}$ & No & 0 & No & Sí & $\begin{array}{l}\text { Vancomicina, } \\
\text { meropenem, } \\
\text { amikacina, } \\
\text { posteriormente solo } \\
\text { vancomicina }\end{array}$ & 10 & Vivo \\
\hline & $3 / \mathrm{M}$ & $\mathrm{LLA}^{\mathrm{f}}$ & $\begin{array}{l}\text { QT fase de } \\
\text { consolidación }\end{array}$ & Endocarditis & 20 & Sí & Sí & $\begin{array}{l}\text { Vancomicina, } \\
\text { piperacilina-tazobactam, } \\
\text { posteriormente } \\
\text { vancomicina y } \\
\text { ceftriaxona }\end{array}$ & 38 & Vivo \\
\hline 2020/México & $2 / \mathrm{M}$ & $\begin{array}{c}\text { Hepatoblastoma } \\
\text { epitelial PRETEXT } \\
3 \text { estadio III }\end{array}$ & $\begin{array}{l}\text { QT doxorrubicina, } \\
\text { cisplatino }\end{array}$ & No & 0 & Sí & No & $\begin{array}{l}\text { Levofloxacino, } \\
\text { vancomicina }\end{array}$ & 7,12 & Vivo \\
\hline 2021/México & $5 / F$ & LLA & $\begin{array}{l}\text { QT vincristina. } \\
\text { pegaspargasa }\end{array}$ & No & 0 & Sí & No & $\begin{array}{l}\text { Meropenem, } \\
\text { vancomicina }\end{array}$ & 7,10 & Vivo \\
\hline \multicolumn{11}{|c|}{$\begin{array}{l}\text { CVC = catéter venoso central; } F=\text { femenino; } L L A=\text { leucemia linfoblástica aguda; } L M A=\text { leucemia mieloide aguda; } M=\text { masculino; NE = no especificado; PRETE } \\
\text { quimioterapia; RPM = ruptura prematura de membranas; RT = radioterapia; } S D G=\text { semanas de gestación; } S N C=\text { sistema nervioso central; TCHP = trasplante d } \\
\text { WBC = conteo total de leucocitos. } \\
\text { a Tratamiento exitoso de meningitis con vancomicina y ceftriaxona por } 21 \text { días. } \\
\text { ' Fallece } 5 \text { meses después por complicaciones de meningitis por } R \text {. mucilaginosa. } \\
\text { ' Ingresó por úlcera gastroesofágica, posteriormente presentó deterioro hemodinámico y ventilatorio. } \\
\text { d Presentó bacteriemia por Staphylococcus aureus } 5 \text { días antes de bacteriemia por } R \text {. mucilaginosus, al día +16 presentó enfermedad por citomegalovirus (CMV). } \\
\text { e Posterior al tratamiento inicial desarrollan meningitis por } R \text {. mucilaginosa. } \\
\text { f Endocarditis con resolución tras } 6 \text { semanas de tratamiento, sin evidencia de vegetaciones al finalizar. }\end{array}$} \\
\hline
\end{tabular}




\begin{tabular}{|c|c|}
\hline Variable & $\mathrm{n}(\%)$ \\
\hline Edad en años, media [rango] & $7.2[0-17]$ \\
\hline \multicolumn{2}{|l|}{ Género } \\
\hline Masculino & $22(71)$ \\
\hline Femenino & $9(29)$ \\
\hline \multicolumn{2}{|l|}{ Comorbilidad } \\
\hline Leucemia aguda & $21(67)$ \\
\hline Leucemia linfoblástica aguda & $14(45)$ \\
\hline Leucemia mieloide aguda & $6(19)$ \\
\hline Leucemia aguda no especificada & $1(3)$ \\
\hline Tumor maligno sólido & $7(23)$ \\
\hline Otro & $3(10)$ \\
\hline \multicolumn{2}{|l|}{ Estado inmune } \\
\hline Quimioterapia & $18(58)$ \\
\hline Radioterapia & $2(6)$ \\
\hline Esteroide & $3(10)$ \\
\hline Cualquiera de los anteriores & $19(61)$ \\
\hline \multirow{3}{*}{\multicolumn{2}{|c|}{$\begin{array}{l}\text { Eventos de complicaciones } \\
\text { Pacientes con complicaci } \\
\text { Complicaciones }\end{array}$}} \\
\hline & \\
\hline & \\
\hline Neumonía & $9(29)$ \\
\hline Infección asociada a CVC & $5(16)$ \\
\hline Meningitis & $5(16)$ \\
\hline Choque séptico & $5(16)$ \\
\hline Sepsis & $2(6)$ \\
\hline Ectima gangrenoso & $1(3)$ \\
\hline Celulitis & $1(3)$ \\
\hline Infección de vías urinarias & $1(3)$ \\
\hline Endocarditis & $1(3)$ \\
\hline \multicolumn{2}{|l|}{ Factores de riesgo } \\
\hline \multicolumn{2}{|l|}{ Neutropenia } \\
\hline Severa RAN $<500$ & $21(68)$ \\
\hline Profunda RAN $<100$ & $11(35)$ \\
\hline Sin neutropenia & $2(6)$ \\
\hline No especificado & $8(26)$ \\
\hline \multicolumn{2}{|l|}{ Mucositis } \\
\hline Mucositis & $9(29)$ \\
\hline Sin mucositis & $16(52)$ \\
\hline No especificado & $6(19)$ \\
\hline \multicolumn{2}{|l|}{ Portador de CVC } \\
\hline Sí & $21(68)$ \\
\hline No & $7(23)$ \\
\hline No especificado & $3(10)$ \\
\hline \multicolumn{2}{|l|}{ Tratamiento } \\
\hline Antibiótico en días, ${ }^{\star}$ media [rango] & $11[10-38]$ \\
\hline \multicolumn{2}{|l|}{ Desenlace $^{\star *}$} \\
\hline Mortalidad general & $5(17)$ \\
\hline $\begin{array}{l}\text { Mortalidad en pacientes con complicaciones } \\
(n=19)^{\star * \star}\end{array}$ & $4(22)$ \\
\hline $\begin{array}{l}\text { Mortalidad en pacientes sin complicaciones } \\
(n=12)\end{array}$ & $1(8)$ \\
\hline \multicolumn{2}{|c|}{$\begin{array}{l}\text { CVC = catéter venoso central; RAN = recuento absoluto de neutrófilos. } \\
{ }^{*} \text { En pacientes que sobrevivieron con duración especificada, en } 11 \text { pacientes } \\
\text { de } 31 \text { no se especificó la duración de tratamiento. } \\
{ }^{* *} \text { Porcentaje en relación con } 30 \text { casos, un caso no reportó desenlace. } \\
{ }^{* * *} \text { Un paciente con complicaciones no reportó desenlace. }\end{array}$} \\
\hline
\end{tabular}

a manera de monoterapia o terapia combinada. El uso predominante de vancomicina concuerda con lo descrito por Wang, quien encontró que en infecciones invasivas por Rothia spp, $76.4 \%$ reciben tratamiento con vancomicina, con duración media de 17 días, y un rango de seis a 27 días. ${ }^{5}$ El segundo grupo de fármacos utilizados con mayor frecuencia fueron las cefalosporinas de tercera generación (n $=12 ; 39 \%$ ). No se reportó uso de trimetoprim con sulfametoxazol en ningún caso, y sólo un caso utilizó ampicilina-sulbactam. La media de duración de antibioticoterapia fue de 17.8 días (rango de 10 a 38 días). En nuestro primer caso se administró meropenem más vancomicina durante siete y 12 días respectivamente, mientras que en el segundo caso se utilizó vancomicina más levofloxacino durante siete y 10 días con evolución favorable en ambos.

Para tratamiento antimicrobiano de primera línea se debe tomar en cuenta la variabilidad en los patrones de susceptibilidad, debido a que se ha descrito resistencia frecuente a quinolonas, clindamicina, aminoglucósidos y trimetoprim con sulfametoxazol. Este microorganismo es generalmente sensible a penicilina, ampicilina, cefotaxima, imipenemcilastatina y vancomicina, esta última con susceptibilidad cercana a 95\%. $2,5,7-9,11-13,15,18-20$ De acuerdo con los datos disponibles, se podría considerar a la vancomicina como fármaco de primera línea para el tratamiento de bacteriemias por R. mucilaginosa; sin embargo, se requiere mejor evidencia para emitir una recomendación contundente. $., 8-13,15,19,22-29$

Debido a la poca frecuencia de los casos, no existe consenso en relación con el curso, pronóstico y mortalidad de la enfermedad, se reporta una mortalidad de hasta $36 \%$. 2,5,15,30 Se obtuvo información acerca del desenlace en 30 pacientes de 31 , en los cuales la mortalidad se presentó en $17 \%$, con una tendencia mayor en el grupo de pacientes con complicaciones asociadas (casos con complicaciones $22 \%$ vs $8 \%$ sin complicaciones). De tal forma que la mortalidad por $R$. mucilaginosa en niños con inmunocompromiso se encuentra entre los valores reportados para bacteriemias por otros cocos grampositivos, como lo describe Regis y colaboradores, en pacientes adultos con neutropenia febril y bacteriemia por estafilococos coagulasa negativo que presentaron mortalidad de $4.3 \%$, y por Vydra y colaboradores de $20 \%$ en bacteriemias por Enterococcus faecium en pacientes pediátricos postrasplantados a 28 y 30 días. ${ }^{31,32}$

La incidencia de bacteriemias en pacientes hematooncológicos por Rothia mucilaginosa es un 
Rev Latin Infect Pediatr. 2021; 34 (4): 193-202

asunto de creciente relevancia que representa un reto diagnóstico y terapéutico ante la falta de guías de tratamiento. Es necesario hacer hincapié en la elaboración de estrategias de manejo para esta etiología, puesto que al no ser considerado como un agente causal frecuente, la importancia pudiera subestimarse y consecuentemente retrasar un abordaje oportuno y el inicio de tratamiento adecuado. Dentro de las opciones terapéuticas se recomienda el inicio de vancomicina como fármaco de primera línea; sin embargo, se requiere un incremento en la calidad de la evidencia para emitir una recomendación contundente.

\section{REFERENCIAS}

1. Collins MD, Hutson RA, Baverud V, Falsen E. Characterization of a Rothia-like organism from a mouse: description of Rothia nasimurium sp. nov. and reclassification of Stomatococcus mucilaginosus as Rothia mucilaginosa comb. nov. Int J Syst Evol Microbiol. 2000; 50 Pt 3: 1247-1251.

2. Poyer F, Friesenbichler W, Hutter C, Pichler H, Dworzak M, Peters $C$ et al. Rothia mucilaginosa bacteremia: A 10-year experience of a pediatric tertiary care cancer center. Pediatr Blood Cancer. 2019; 66 (7): e27691.

3. Silva FO. Rothia mucilaginosa. retrato microbiológico. Rev Chil Infect. 2008; 25 (1): 29.

4. Trivedi MN, Malhotra P. Rothia prosthetic knee joint infection. J Microbiol Immunol Infect. 2015; 48 (4): 453-455.

5. Wang JY, Brossard J, Cellot S, Dix D, Feusner J, Johnston DL et al. Invasive Rothia infections in children with acute myeloid leukemia: a report from the Canadian infections in AML research group. Pediatr Hematol Oncol. 2016; 33 (5): 277-281.

6. Mikulska M, Viscoli C. Gram-positive bacterial infections after haematopoietic stem cell or solid organ transplantation. In: Ljungman P, Snydman D, Boeckh M (eds). Transplant infections. Cham: Springer; 2016. Available in: https://doi. org/10.1007/978-3-319-28797-3_20

7. Rubin SJ, Lyons RW, Murcia AJ. Endocarditis associated with cardiac catheterization due to a Gram-positive coccus designated Micrococcus mucilaginosus incertae sedis. J Clin Microbiol. 1978; 7 (6): 546-549.

8. Treviño M, García-Zabarte A, Quintás A, Varela E, LópezPaz JM, Jato A et al. Stomatococcus mucilaginosus septicemia in a patient with acute lymphoblastic leukaemia. Eur J Clin Microbiol Infect Dis. 1998; 17 (7): 505-507.

9. Chavan RS, Pannaraj PS, Luna RA, Szabo S, Adesina $A$, Versalovic $J$ et al. Significant morbidity and mortality attributable to Rothia mucilaginosa infections in children with hematological malignancies or following hematopoietic stem cell transplantation. Pediatr Hematol Oncol. 2013; 30 (5): 445-454.

10. Ochi F, Tauchi H, Moritani K, Yonezawa S, Miyamoto H, Suemori K et al. Rothia mucilaginosa infection in a child with acute lymphoblastic leukemia. Pediatr Blood Cancer. 2017; 64 (1): 205-206

11. Gutierrez V, Cerda C, Alcantara A, Clavarie X. Rothia mucilaginosa, an important cause of invasive disease in children with leukemia: report of 2 cases and review of the literature. Authorea [Internet]. 2020. Available in: https://www.authorea.com/doi/full/10.22541/ au.159284826.61673369

12. Park MK, Khan J, Stock F, Lucey DR. Successful treatment of Stomatococcus mucilaginosus meningitis with intravenous vancomycin and intravenous ceftriaxone. Clin Infect Dis. 1997; 24 (2): 278.

13. Bayhan C, Karadag Oncel E, Cengiz AB, Oksuz AB, Aydin GB. Bacteriemia causada por Rothia mucilaginosa después de neumonía en un paciente con trasplante de células progenitoras hematopoyéticas. Arch Argent Pediatr. 2016; 114 (5): 343-345.

14. Khan ST, Ahamed M, Musarrat J, Al-Khedhairy AA. Antibiofilm and antibacterial activities of zinc oxide nanoparticles against the oral opportunistic pathogens Rothia dentocariosa and Rothia mucilaginosa. Eur J Oral Sci. 2014; 122 (6): 397403.

15. Henwick S, Koehler M, Patrick CC. Complications of bacteremia due to Stomatococcus mucilaginosus in neutropenic children. Clin Infect Dis. 1993; 17 (4): 667671.

16. Morgan EA, Henrich TJ, Jarell AD, Shieh WJ, Zaki SR, Marty FM et al. Infectious granulomatous dermatitis associated with Rothia mucilaginosa bacteremia: a case report. Am J Dermatopathol. 2010; 32 (2): 175-179.

17. Clinical and Laboratory Standards Institute (CLSI). Methods for antimicrobial dilution and disk susceptibility testing of infrequently isolated or fastidious bacteria. CLSI guideline M45. 3rd edition. Wayne, Pennsylvania: CLSI; 2015.

18. McWhinney PH, Kibbler CC, Gillespie SH, Patel S, Morrison D, Hoffbrand AV et al. Stomatococcus mucilaginosus: an emerging pathogen in neutropenic patients. Clin Infect Dis. 1992; 14 (3): 641-646.

19. Bruminhent J, Tokarczyk MJ, Jungkind D, DeSimone JA Jr. Rothia mucilaginosa prosthetic device infections: a case of prosthetic valve endocarditis. J Clin Microbiol. 2013; 51 (5): 1629-1632.

20. Ramos JM, Mateo I, Vidal I, Rosillo EM, Merino E, Portilla J. Infection due to Rothia mucilaginosa. A respiratory pathogen? Enferm Infecc Microbiol Clin. 2014; 32 (5): 306309.

21. Goldman M, Chaudhary UB, Greist A, Fausel CA. Central nervous system infections due to Stomatococcus mucilaginosus in immunocompromised hosts. Clin Infect Dis. 1998; 27 (5): 1241-1246.

22. Weinblatt ME, Sahdev I, Berman M. Stomatococcus mucilaginosus infections in children with leukemia. Pediatr Infect Dis J. 1990; 9 (9): 678-679.

23. Ascher DP, Zbick C, White C, Fischer GW. Infections due to Stomatococcus mucilaginosus: 10 cases and review. Rev Infect Dis. 1991; 13 (6): 1048-1052.

24. Langbaum M, Eyal FG. Stomatococcus mucilaginosus septicemia and meningitis in a premature infant. Pediatr Infect Dis J. 1992; 11 (4): 334-335.

25. Souillet G, Chomarat M, Barbé G, Balouck N, Ploton C, Philippe N. Stomatococcus mucilaginosus meningitis in a child with leukemia. Clin Infect Dis. 1992; 15 (6): 1045.

26. Andstrom E, Bygdeman S, Ahlén S, Heimdal A, Nystrom B. Stomatococcus mucilaginosus septicemia in two bone marrow transplanted patients. Scand J Infect Dis. 1994; 26 (2): 209-214. 
27. Vaccher S, Cordiali R, Osimani P, Manso E, de Benedictis FM. Bacteremia caused by Rothia mucilaginosa in a patient with Shwachman-Diamond syndrome. Infection. 2007; 35 (3): 209-210.

28. Mustafa MM, Carlson LR, Krisher K. Stomatococcus mucilaginosus fatal sepsis in a child with leukemia. Pediatr Infect Dis J. 1993; 12 (9): 784-786.

29. Kaufhold A, Reinert RR, Kern W. Bacteremia caused by Stomatococcus mucilaginosus: report of seven cases and review of the literature. Infection. 1992; 20 (4): 213-220.

30. Rizvi M, Fatima N, Shukla I, Afzal K. Stomatococcus mucilaginosus meningitis in a healthy 2 -month-old child. $\mathrm{J}$ Med Microbiol. 2008; 57 (Pt 3): 382-383.

31. Vydra J, Shanley RM, George I, Ustun C, Smith AR, Weisdorf DJ et al. Enterococcal bacteremia is associated with increased risk of mortality in recipients of allogeneic hematopoietic stem cell transplantation. Clin Infect Dis. 2012; 55 (6): 764-770.

32. Rosa RG, Dos Santos RP, Goldani LZ. Mortality related to coagulase-negative staphylococcal bacteremia in febrile neutropenia: A cohort study. Can J Infect Dis Med Microbiol. 2014; 25 (1): e14-e17.

33. Ascher DP, Bash MC, Zbick C, White C. Stomatococcus mucilaginosus catheter-related infection in an adolescent with osteosarcoma. South Med J. 1991; 84 (3): 409-410.

\section{Conflicto de intereses: Sin conflicto de intereses.}

Correspondencia:

Denisse Natalie Vaquera Aparicio

E-mail: dra.denissevaquera@gmail.com

\section{Anexo 1: Estrategia de búsqueda con términos MeSH en inglés y español.}

("bacteriemia" OR "bacteremia" or "bacteraemia" OR "blood culture" OR "septicemia" OR "septicaemia" OR "sepsis" OR "septic shock" OR "catheter-related bloodstream infection" OR "catheter-related sepsis" OR "central line bloodstream infection" OR "intravascular infection" OR "primary bloodstream infection" OR "meningitis" OR "septic arthritis" OR "endocarditis" OR "pericarditis" OR "peritonitis" OR "pyelonephritis" OR "colitis" OR "mucositis" OR "pneumonia" OR "soft tissue infection" OR "cellulitis" OR "stomatitis" OR "periodontitis" OR "periodontal disease" OR "osteomyelitis" OR "bone infection" OR "joint infection" OR "endophtalmitis" OR "eye infection") AND ("rothia" OR "rothia sp" OR "rothia spp" OR "Rothia mucilaginosa" OR "R. mucilaginosa" OR "Stomatococcus mucilaginosus" OR "s. mucilaginosus" OR "stomatococcus sp" OR "stomatococcus spp" OR "Micrococcus mucilaginosus" OR "m. mucilaginosus") AND ("immunocompromised" OR "immunocompromise" OR "immunocompromised-host" OR "immunocompromised patient" OR "immunosuppressor" OR "immunosuppressive" OR "neutropenic" OR "neutropenia" OR "steroids" OR "cancer" OR "neoplasm" OR "malignancy" OR "immunodeficiency" OR "chemotherapy" OR "chemotherapeutic" OR "radiotherapy" OR "radiotherapeutic" OR "leukemia" OR "bone marrow transplant" OR "stem cell transplant" OR "hematopoietic stem cell transplant" OR "transplant-associated infection") 